\title{
Cyclooctyne-based reagents for uncatalyzed click chemistry: A computational survey $\uparrow$
}

\author{
Kimberly Chenoweth, \$ David Chenoweth§ and William A. Goddard III*
}

\author{
Received 12th June 2009, Accepted 16th September 2009 \\ First published as an Advance Article on the web 9th November 2009 \\ DOI: 10.1039/b911482c
}

With the goal of identifying alkyne-like reagents for use in click chemistry, but without Cu catalysts, we used B3LYP density function theory (DFT) to investigate the trends in activation barriers for the 1,3-dipolar cycloadditions of azides with various cyclooctyne, dibenzocyclooctyne, and azacyclooctyne compounds. Based on these trends, we find monobenzocyclooctyne-based reagents that are predicted to have dramatically improved reactivity over currently employed reagents.

\section{Introduction}

An explosion of techniques has energized the bioorthogonal ligation chemistry field, with new methodologies involving oxime and hydrazone formation, Staudinger ligation, and azide-alkyne [3+2] cycloaddition. ${ }^{1}$ Although many areas of chemistry benefit from click chemistry, the toxicity of the $\mathrm{Cu}$ catalyst in the [3+2] azide-alkyne cycloaddition has prohibited in vivo applications. ${ }^{1 \mathrm{~d}, 1 \mathrm{e}}$ Thus, room-temperature labeling reagents that avoid using copper are highly desirable.

Similarly, click reactions provide a means for unique surface functionalization and labelling strategies with application in nanotechnology and surface science. For example, the Heath group and others have developed strategies for functionalizing $\mathrm{Si}$ and Au surfaces with azides, which could be attached to alkyneterminated labels or rotaxanes for sensors or nanomechanical systems. ${ }^{2}$ Here it would be most useful to avoid the requirement of copper catalysts while accelerating the reactions to be fast at room temperature.

Recently, the Bertozzi group ${ }^{3}$ demonstrated a new strain-release cyclooctyne labelling reagent $(\mathbf{1}$, Scheme 1$)$ that proceeds in the absence of a $\mathrm{Cu}$ catalyst at physiological temperatures, although with a $c a$. 52-fold reduction in reaction rate. ${ }^{1 a}$ The increased reactivity of cyclooctyne toward [3+2] cycloaddition has been attributed to strain release, and estimates of the cyclooctyne ring strain range from $10-19 \mathrm{kcal} / \mathrm{mol}^{4}{ }^{4}$ The ground-state destabilizing effect of the triple bond is thought to drive the bioorthogonal ligation reactions (e.g. attachment of chemical reporter molecules) in the absence of a catalyst; however, this has been challenged with the concept of dipole distortion. ${ }^{4 \mathrm{~d}, 9}$ Bertozzi demonstrated that gem-difluoro substitution $\alpha$ to the alkyne leads to a reaction rate 63 times faster than the simple cyclooctyne, which has been attributed to the greater LUMO-lowering effect of the

Materials and Process Simulation Center, Division of Chemistry and Chemical Engineering, California Institute of Technology, Pasadena, California, 91125, USA. E-mail: wag@wag.caltech.edu; Fax: +1 (626)585-0918; Tel: +1 (626)395-2731

$\dagger$ Electronic supplementary information (ESI) available: Absolute energies and cartesian coordinates. See DOI: 10.1039/b911482c

$\$$ Current address: Department of Chemistry, Smith College, Northampton, Massachusetts 01063, USA.

$\S$ Current address: Department of Chemistry, Massachusetts Institute of Technology, 77 Massachusetts Avenue, Cambridge, MA 02139, USA

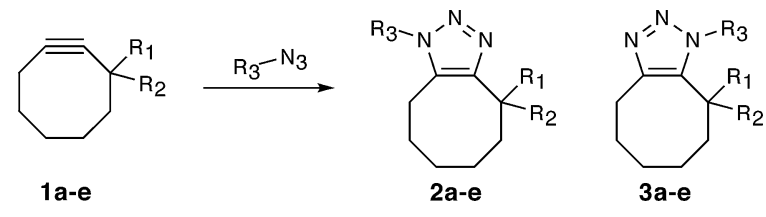

Scheme 1 Strain-release azide-alkyne cycloaddition reactions.

fluoro substituent. ${ }^{3 c}$ In addition, dibenzocyclooctyne reagents have allowed visualization of glycoconjugates in living cells using confocal microscopy while a difluorinated cyclooctyne derivative has proven useful for investigating in vivo glycan trafficking dynamics. ${ }^{3 c, 5}$

First, we investigated the best current bioorthogonal cyclooctyne-based labelling reagents to gain insight into reactivity patterns associated with electronic, steric, and strain effects by assessing trends in the activation energies, thus providing a basis of comparison for potential monobenzocyclooctyne reagents. In addition, we investigated the decomposition mechanism observed for a monofluorinated cyclooctyne reagent. Next, we assessed dibenzocyclooctyne- and azacyclooctyne-based reagents and compared the calculated barriers to the cyclooctynes. Finally, we investigated new monobenzocyclooctynes and compared the calculated barriers to that of cyclooctyne and dibenzocyclooctyne reagents. To date, these monocyclooctynes have not been used in bioorthogonal ligation chemistry but provide improved reactivity compared to previously reported cyclooctyne-based reagents.

\section{Computational details}

All calculations were performed using the B3LYP hybrid DFT functional ${ }^{6}$ with the $6-311 \mathrm{G}^{* *}$ basis set $^{7}$ as implemented in the Jaguar 6.5 software package. ${ }^{8}$ Vibrational frequencies have been calculated at all stationary points to obtain zero-point energies (ZPE) and free energies. All stationary points have been identified as local minima (zero imaginary frequencies) or transition states (TS) (one imaginary frequency). We should emphasize that the interest here is in identifying the cyclooctyne reagent with the lowest barrier for cycloaddition to an azide (click chemistry). These DFT calculations may overestimate absolute reaction energies by a few $\mathrm{kcal} / \mathrm{mol}^{4 \mathrm{~d}}$ 
Table 1 Activation energy (in $\mathrm{kcal} / \mathrm{mol}$ ) for transition states TS1 and TS2 in Fig. 1. Best is 1e, confirmed experimentally ${ }^{3 c}$

\begin{tabular}{|c|c|c|c|c|c|c|}
\hline \multirow[b]{2}{*}{ Reactant } & \multirow[b]{2}{*}{$\mathrm{R}_{1}$} & \multirow[b]{2}{*}{$\mathrm{R}_{2}$} & \multicolumn{2}{|l|}{$\Delta E^{*}$} & \multicolumn{2}{|l|}{$\Delta G^{\ddagger}$} \\
\hline & & & TS1 & TS2 & TS1 & TS2 \\
\hline $1 \mathrm{a}$ & $\mathrm{H}$ & $\mathrm{H}$ & 12.8 & - & 24.9 & - \\
\hline $1 b$ & $\mathrm{Me}$ & $\mathrm{H}$ & 13.7 & 13.6 & 26.1 & 26.1 \\
\hline $1 c$ & $\mathrm{Me}$ & $\mathrm{F}$ & 13.7 & 11.3 & 26.1 & 24.7 \\
\hline 1d & $\mathrm{H}$ & $\mathrm{F}$ & 12.6 & 10.5 & 24.6 & 23.4 \\
\hline $1 \mathrm{e}$ & $\mathrm{F}$ & $\mathrm{F}$ & 12.1 & 9.3 & 24.6 & 22.1 \\
\hline
\end{tabular}

\section{Results and discussion}

\section{Cyclooctyne-based reagents}

We first studied a series of model fluorinated cyclooctynes analogous to the bioorthogonal reagents developed in the Bertozzi group. To determine how various substituents affect the reactivity of cyclooctyne with methyl azide, we calculated the activation energies (Table 1) for formation of both triazole regioisomers for a range of substituents (Fig. 1). We find that methyl substitution adjacent to the triple bond of cyclooctyne (1b), leads to a slight increase in the activation barrier; however, fluorination at the $\alpha$-position (1c, $\mathbf{1 d}, \mathbf{1 e})$ leads to a consistent decrease in the activation energy as the number of fluorines increase. In addition, changing from the difluorinated cyclooctyne (1e) to a mono-fluorinated cyclooctyne (1d) increases the azide-alkyne cycloaddition barrier by $\Delta \Delta G^{\ddagger}=1.3 \mathrm{kcal} / \mathrm{mol}$ for TS2, which is consistent with the factor of $\sim 10$ decrease in reaction rates observed experimentally. ${ }^{3 \mathrm{c}}$ The reaction of gem-difluorocyclooctyne (1e) with methyl azide leads to a transition state barrier for 1e-TS2 of $22.1 \mathrm{kcal} / \mathrm{mol}$, which is $\Delta \Delta G^{\ddagger}=2.8 \mathrm{kcal} / \mathrm{mol}\left(\Delta \Delta E^{\ddagger}=3.5 \mathrm{kcal} / \mathrm{mol}\right)$ lower in energy than reaction with cyclooctyne (1a), leading to a 106-fold rate enhancement. This is consistent with the observed 63 -fold rate enhancement (from $1.2 \times 10^{-3} \mathrm{M}^{-1} \mathrm{~s}^{-1}$ to $7.6 \times 10^{-2} \mathrm{M}^{-1} \mathrm{~s}^{-1}$ ) for similar substrates. ${ }^{3}$ [The slight disparity in rate and regioselectivity (63 vs. 106) may be attributed to the solvation effects ${ }^{9 a}$ and the difference between the benzyl azide used in experiments compared to our use of methyl azide as a model system.] Our calculated activation barriers are consistent with more recent theoretical

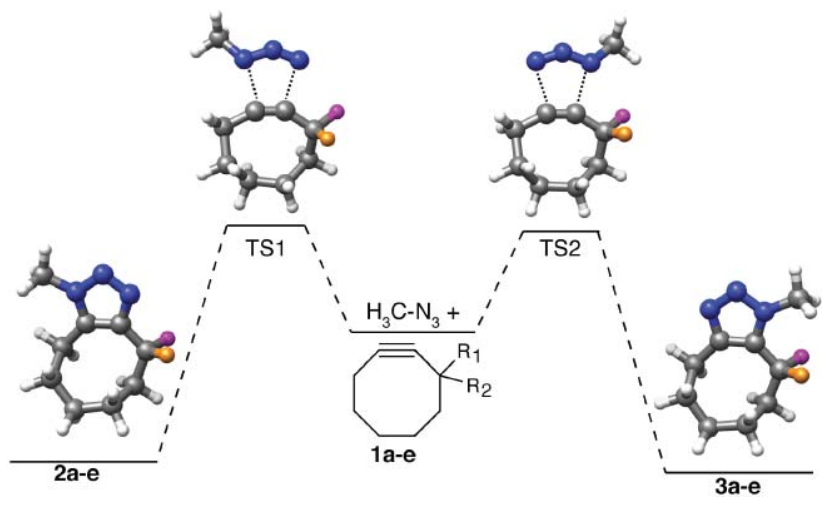

Fig. 1 Azide-cyclooctyne reaction pathways. $\mathrm{R}_{1}$ (pink) and $\mathrm{R}_{2}$ (orange) are defined in Table 1. studies for reaction with methyl azide, and the trends in the barriers are consistent with reaction with phenyl azide..$^{4 \mathrm{~d}, 9}$

\section{Decomposition of the product (2c) from cyclooctyne 1c}

Interestingly, the Bertozzi group observed that when reacting reagents analogous to $1 \mathrm{c}$ with azides one of the triazole regioisomers underwent decomposition where the other isomer was stable. ${ }^{2 b}$ Experimental observations have indicated that fluorination at a tertiary carbon position $\alpha$ to the alkyne in a cyclooctynebased reagent can enhance the elimination processes. ${ }^{2 b}$ To gain insight into the experimentally observed regioisomeric decomposition of the triazole products formed from cycloaddition of the monofluorinated cyclooctyne $\mathbf{1 c}$, we propose a reaction intermediate to the decomposition reaction and a mechanism based on steric $\mathrm{A}^{1,3}$ strain that precludes decomposition of product isomer 3c (Fig. 2). This suggests the possibility of a resonance-assisted (hyperconjugation involving the $\mathrm{NCH}_{3}$ lone pair) steric-free pathway, as a limiting case, that proceeds through a carbocation intermediate (2c-cation), formed via fluorine elimination, to the decomposition product $\mathbf{2 c - O H}$ resulting from $\mathrm{S}_{\mathrm{N}} 1$-type attack by water, with the actual mechanism probably lying somewhere on the continuum between the $\mathrm{S}_{\mathrm{N}} 1$ and $\mathrm{S}_{\mathrm{N}} 2$ extremes. We optimized the structures for the carbocation intermediates in the decomposition pathway (see Fig. 2), leading to a DFT energy for carbocation 3c-cation higher by $\Delta E=12.7 \mathrm{kcal} / \mathrm{mol}$ than that of 2 c-cation, due primarily to $\mathrm{A}^{1,3}$ strain. This precludes full resonance stabilization by the triazole ring, as shown by the resulting angles in Fig. 2. This energetic difference in carbocations explains the decomposition of $\mathbf{2 c}$ versus the stability of $\mathbf{3 c}$, providing insight into the importance of the appropriate substitution $\alpha$ to the alkyne and $\mathrm{A}^{1,3}$ strain for future reagent design.

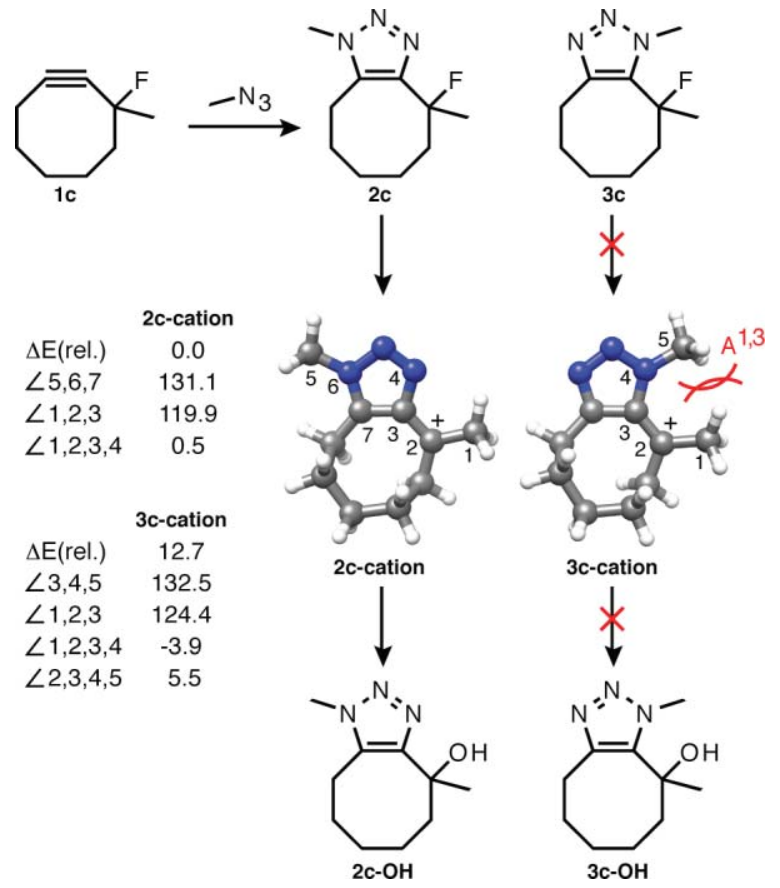

Fig. 2 Decomposition pathway for $\alpha$-monofluorinated cyclooctyne-based reagents. 


\section{Azacyclooctyne-based reagent}

Next, we evaluated the barriers for azide-alkyne cycloaddition for a heterocyclic and heteroatom-substituted cyclooctyne-based reagent (4, Fig. 3). Interest in this reagent lies in the experimental observation that it improves sensitivity of azide detection due to its superior polarity and water solubility due to the presence of the methoxy groups. ${ }^{3 e}$ Experimentally, the azacyclooctyne, 6,7-dimethoxyazacyclooct-4yne (DIMAC), was found to provide a rate enhancement of 2.5-fold over 1a for the 1,3'-dipolar cycloaddition reaction with benzyl azide (from $1.2 \times 10^{-3} \mathrm{M}^{-1} \mathrm{~s}^{-1}$ to $\left.3.0 \times 10^{-3} \mathrm{M}^{-1} \mathrm{~s}^{-1}\right) .{ }^{3 e}$ Our calculations find a similar increase in rate for a model DIMAC compound (4) over cyclooctyne (1a) with a decrease in barrier height of $\Delta \Delta G^{\ddagger}=0.3 \mathrm{kcal} / \mathrm{mol}$, corresponding to a rate enhancement of $\sim 2$.
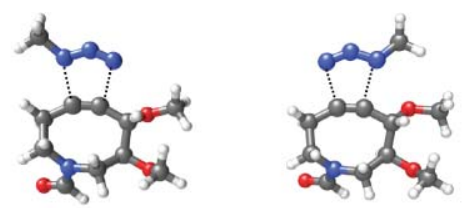

$\Delta \mathrm{E}^{\ddagger}=13.1\left(\Delta \mathrm{G}^{\ddagger}=25.0\right)$

$\Delta \mathrm{E}^{\ddagger}=11.4\left(\Delta \mathrm{G}^{\ddagger}=24.6\right)$
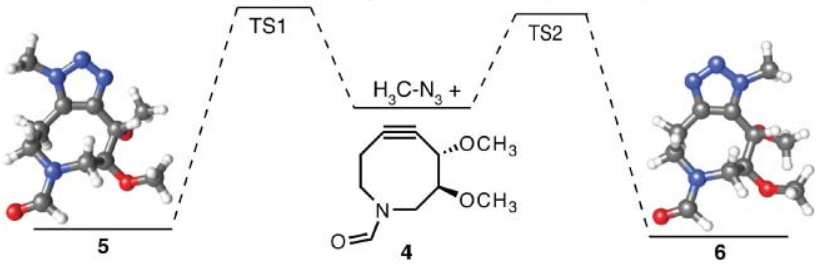

Fig. 3 Azide-azacyclooctyne cycloaddition reaction pathway.

\section{Dibenzocyclooctyne-based reagents}

Another class of reagents investigated for use as bioorthogonal reagents is the dibenzocyclooctynes. In order to compare these systems to the nonbenzoid cyclooctyne-based reagents, we calculated the activation energies for cycloaddition with methyl azide (Fig. 4). Structure $7 \mathbf{f}$ with $\mathrm{R}_{4}=\mathrm{R}_{5}=\mathrm{H}$ is comparable to the reagent with $\mathrm{R}_{4}=\mathrm{OH}$ and $\mathrm{R}_{5}=\mathrm{H} .{ }^{5}$ We find that the cycloaddition barrier for $7 \mathbf{f}$ is $12.3 \mathrm{kcal} / \mathrm{mol}$ (Table 2), which is $0.5 \mathrm{kcal} / \mathrm{mol}$ lower than for cyclooctyne 1a (Table 1). It has been proposed that the biaryl substitution would increase ring strain and provide conjugation

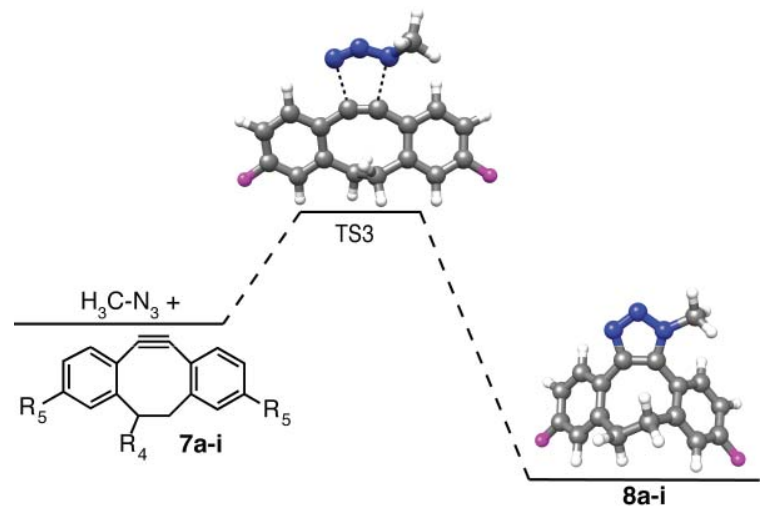

Fig. 4 Azide-dibenzocyclooctyne cycloaddition reaction pathways. $R_{4}$ is hydrogen, and $R_{5}$ (pink) is defined in Table 2 .
Table 2 Activation energy ( $\mathrm{kcal} / \mathrm{mol})$ for transition state TS3 in Fig. 2

\begin{tabular}{llll}
\hline Reactant & $\mathrm{R}_{5}$ & $\Delta E^{\ddagger}$ & $\Delta G^{\ddagger}$ \\
\hline $\mathbf{7 a}$ & $\mathrm{NH}_{2}$ & 12.2 & 25.5 \\
$\mathbf{7 b}$ & $\mathrm{OMe}$ & 11.6 & 24.9 \\
$\mathbf{7 c}$ & $\mathrm{OEt}$ & 11.7 & 25.1 \\
$\mathbf{7 d}$ & $\mathrm{N}\left(\mathrm{CH}_{3}\right)_{2}$ & 12.2 & 25.7 \\
$\mathbf{7 e}$ & $\mathrm{H}$ & 12.3 & 25.5 \\
$\mathbf{7 f}$ & $\mathrm{F}$ & 11.8 & 25.2 \\
$\mathbf{7 g}$ & $\mathrm{Cl}$ & 11.7 & 25.0 \\
$\mathbf{7 h}$ & $\mathrm{NO}_{2}$ & 11.2 & 25.1 \\
$\mathbf{7 i}$ & $\mathrm{CN}$ & 11.3 & 24.7 \\
\hline
\end{tabular}

with the alkyne, resulting in improved reactivity. ${ }^{5}$ However, we find from our calculations that the resulting steric interaction between the methyl group on the azide and the hydrogen of the phenyl group ( $\mathrm{A}^{1,3}$ strain) competes directly with the activating effects of the aryl ring, resulting in hampered reactivity compared to Bertozzi's fluorinated cyclooctyne-based reagents. The rate constants for dibenzocyclooctynol were reported ${ }^{5}$ to have a rate acceleration of approximately three orders of magnitude over that of cyclooctyn-ol. However, our calculations find very similar barriers between cyclooctyne and a dibenzocyclooctyne (e.g., with cyclooctyne, leading to a factor of 2 enhancement). The origin of this discrepancy might be related to solvent effects, reaction conditions, the use of benzyl azide instead of methyl azide, or differences in the substrates. Based on our calculations and results in the literature, ${ }^{4 \mathrm{~d}, 9}$ the origin of this discrepancy might be related to the specific experimental reaction conditions, and it would be useful for the rates of the various cyclooctyne reagents to be examined in a thorough experimental study. In addition, we find that para-substitution on the phenyl group has very little inductive effect and does not significantly reduce the activation barriers (Table 2).

\section{Benzocyclooctyne-based reagents}

Based on these new insights into azide-alkyne cycloadditions, we propose new cyclooctyne reagents bearing a single aryl substituent (Fig. 5) under the hypothesis that decreased $\mathrm{A}^{1,3}$ strain, transannular hybridization-induced ring strain, and electronwithdrawing substituents could yield a superior reagent with improved reactivity. We find that the position of the phenyl group with respect to the alkyne has a significant effect on the reactivity toward cycloaddition. Thus fusing a benzene ring to carbon 3-4 of the cyclooctyne system 9-TS1 and 9-TS2 (Fig. 5) leads to the largest barriers to cycloaddition, 25.3 and $24.9 \mathrm{kcal} / \mathrm{mol}$ respectively. Moving the aryl fusion to the 4-5 position of the cyclooctyne system decreases the barrier heights for 10-TS1 and 10-TS2 to 23.3 and $23.5 \mathrm{kcal} / \mathrm{mol}$, respectively. This decrease in activation energy and the energetic switch in regioisomeric activation energies is presumably due to superposition of reduced $\mathrm{A}^{1,3}$ interactions and decreased electronic effects from the aryl group. Fusing the aryl group at the 5-6 carbon position of the cyclooctyne ring 11-TS further decreases the barrier for cycloaddition with methyl azide to $22.1 \mathrm{kcal} / \mathrm{mol}$, making it competitive with some of the best fluorinated cyclooctyne systems (1e with $22.1 \mathrm{kcal} / \mathrm{mol}$ ). In addition, we find that a 6 -membered aromatic ring in this position provides increased angle compression, leading to improved reactivity, whereas a 5-membered aromatic ring 


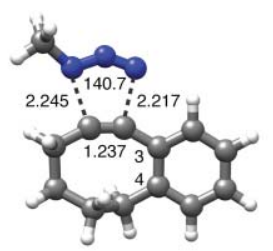

9-TS1

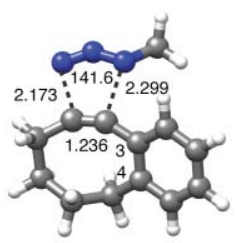

9-TS2

$\Delta \mathrm{E}^{\ddagger}=12.0\left(\Delta \mathrm{G}^{\ddagger}=24.9\right)$
$\Delta \mathrm{E}^{\ddagger}=12.7\left(\Delta \mathrm{G}^{\ddagger}=25.3\right)$

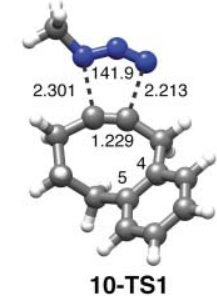

$\Delta \mathrm{E}^{\ddagger}=11.0\left(\Delta \mathrm{G}^{\ddagger}=23.3\right)$

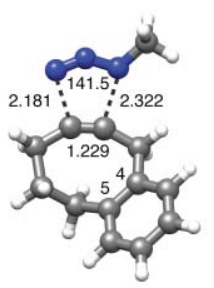

10-TS2

$\Delta \mathrm{E}^{\ddagger}=11.3\left(\Delta \mathrm{G}^{\ddagger}=23.5\right)$

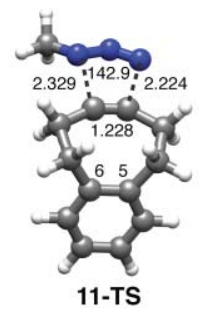

$\Delta \mathrm{E}^{\ddagger}=10.0\left(\Delta \mathrm{G}^{\ddagger}=22.1\right)$

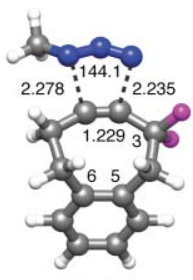

12-TS1

$\mathrm{E}^{\ddagger}=9.1\left(\Delta \mathrm{G}^{\ddagger}=21.7\right)$

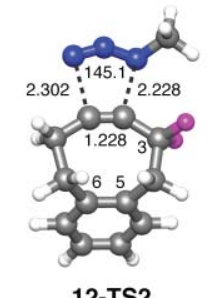

12-TS2

$\Delta \mathrm{E}^{\ddagger}=6.7\left(\Delta \mathrm{G}^{\ddagger}=19.7\right)$
Fig. 5 Azide-benzocyclooctyne (BC8y) cycloaddition transition-state structures and activation barriers in $\mathrm{kcal} / \mathrm{mol}$. Bond distances are in $\AA$ and angles are in degrees. The best is gem-difluorobenzocyclooctyne, $\mathbf{1 0}$.

results in less angle compression and decreased reactivity (see Fig. $\mathrm{S} 1 \dagger)$. These benzocyclooctynes have been reported to be isolable, but they have not been utilized for copper-free click chemistry. ${ }^{10}$

Combining the positional effects of the aryl substituent and the LUMO lowering electron-withdrawing effects of the gem-difluoro substituent, we find significantly decreased activation energies to $19.7 \mathrm{kcal} / \mathrm{mol}$ for the $\mathbf{1 2}$-TS2 cycloaddition transition state structure shown in Fig. 2 (and $21.7 \mathrm{kcal} / \mathrm{mol}$ for 12-TS1). This is lower than the barrier for $1 \mathrm{e}\left(\Delta \Delta G^{\ddagger}=2.4 \mathrm{kcal} / \mathrm{mol}\right)$, making it 57 times faster, and suggesting a rate of $57 \times\left(7.6 \times 10^{-2} \mathrm{M}^{-1} \mathrm{~s}^{-1}\right)=$ $4.4 \mathrm{M}^{-1} \mathrm{~s}^{-1}$. ${ }^{11}$ Since $1 \mathrm{e}$ has been observed to be 52 times slower than the copper-catalyzed system, the gem-difluorobenzocyclooctyne (12) should exhibit activity comparable to or faster than the copper-catalyzed click reaction. ${ }^{3 c}$ We expect that this new class of benzocyclooctyne reagents will provide a range of stabilities and reactivities to enable room-temperature copper-free click chemistry for applications in biology, nanotechnology, and surface science. In addition, note that the simple benzocyclooctyne $\mathbf{1 1}$ has the same barrier and rate as the best previous copper-free click reagent, gem-difluorocyclooctyne (1e), making it a potentially useful reagent.

This class of benzocyclooctynes takes advantage of the optimal placement of $\mathrm{sp}^{2}$ centers for the enhancement of ring strain and the proper placement of electron-withdrawing substituents for LUMO-lowering effects while avoiding the detrimental effects of $\mathrm{A}^{1,3}$ interactions.

\section{Conclusions}

This exercise demonstrates how modern computational chemistry provides a valuable tool for a priori design of optimal reagents for novel applications in biological systems and materials science, dramatically reducing the number of experiments required to navigate structure-sequence space. In particular, we show that combining transannular hybridization-induced ring strain with proper placement of LUMO-lowering electron-withdrawing substituents, while avoiding deleterious effects of $\mathrm{A}^{1,3}$ interactions, provides guiding principles for designing superior cyclooctynebased reagents with improved reactivity.

\section{References}

1 (a) J. A. Prescher and C. R. Bertozzi, Nat. Chem. Biol., 2005, 1, 13; (b) M. Kohn and R. Breinbauer, Angew. Chem., Int. Ed., 2004, 43, 3106; (c) R. Huisgen, Angew. Chem., Int. Ed. Engl., 1963, 2, 565; (d) V. V. Rostovtsev, L. G. Green, V. V. Fokin and K. B. Sharpless, Angew. Chem., Int. Ed., 2002, 41, 2596; (e) H. C. Kolb, M. G. Finn and K. B. Sharpless, Angew. Chem., Int. Ed., 2001, 40, 2004.

2 (a) R. D. Rohde, H. D. Agnew, W.-S. Yeo, R. C. Bailey and J. R. Heath, J. Am. Chem. Soc., 2006, 128, 9518; (b) N. K. Devaraj, G. P. Miller, W. Ebina, B. Kakaradov, J. P. Collman, E. T. Kool and C. E. D. Chidsey, J. Am. Chem. Soc., 2005, 127, 8600; (c) N. K. Devaraj, P. H. Dinolfo, C. E. D. Chidsey and J. P. Collman, J. Am. Chem. Soc., 2006, 128, 1794.

3 (a) N. J. Agard, J. A. Prescher and C. R. Bertozzi, J. Am. Chem. Soc., 2004, 126, 15046; (b) N. J. Agard, J. M. Baskin, J. A. Prescher, A. Lo and C. R. Bertozzi, ACS Chem. Biol., 2006, 1, 644; (c) J. M. Baskin, J. A. Prescher, S. T. Laughlin, N. J. Agard, P. V. Chang, I. A. Miller, A. Lo, J. A. Codelli and C. R. Bertozzi, Proc. Natl. Acad. Sci. U. S. A., 2007, 104, 16793; (d) S. T. Laughlin, J. M. Baskin, S. L. Amacher and C. R. Bertozzi, Science, 2008, 320, 664; (e) E. M. Sletten and C. R. Bertozzi, Org. Lett., 2008, 10, 3097.

4 (a) G. Wittig and A. Krebs, Chem. Ber., 1961, 94, 3260; (b) R. B. Turner, A. D. Jarrett, P. Goebel and B. J. Mallon, J. Am. Chem. Soc., 1973, 95, 790; (c) J. F. Liebman and A. Greenberg, Chem. Rev., 1976, 76, 311; (d) R. D. Bach, J. Am. Chem. Soc., 2009, 131, 5233.

5 X. Ning, J. Guo, M. A. Wolfert and G. J. Boons, Angew. Chem., Int. Ed., 2008, 47, 2253.

6 A. D. Becke, J. Chem. Phys., 1993, 98, 5648; C. Lee, W. Yang and R. G. Parr, Phys. Rev. B, 1988, 37, 785.

7 R. Krishnan, J. S. Binkley, R. Seeger and J. A. Pople, J. Chem. Phys., $1980,72,650$.

8 (a) Jaguar 6.5, Schrodinger, LLC: Portland, OR, 2005; (b) B. H. Greeley, T. V. Russo, D. T. Mainz, R. A. Friesner, J.-M. Langlois, W. A. Goddard III, R. E. Donnelly and M. N. Ringnalda, J. Chem. Phys., 1994, 101, 4028; (c) D. J. Tannor, B. Marten, R. Murphy, R. A. Friesner, D. Sitkoff, A. Nicholls, M. Ringnalda, W. A. Goddard III and B. Honig, J. Am. Chem. Soc., 1994, 116, 11875.

9 (a) F. Schoenebeck, D. H. Ess, G. O. Jones and K. N. Houk, J. Am. Chem. Soc., 2009, 131, 8121; (b) D. H. Ess, G. O. Jones and K. N. Houk, Org. Lett., 2008, 10, 1633.

10 A. Krebs and J. Wilke, Top. Curr. Chem., 1983, 109, 189.

11 Structures were rendered using UCSF Chimera, Resource for Biocomputing, Visualization, and Informatics, University of California, San Francisco (supported by NIH P41 RR-01081). See: E. F. Pettersen, T. D. Goddard, C. C. Huang, G. S. Couch, D. M. Greenblatt, E. C. Meng and T. E. Ferrin, J. Comput. Chem., 2004, 25, 1605. 